\title{
Dynamic recrystallization induced by plastic deformation at high strain rate in a Monel alloy
}

\author{
Q. Li ${ }^{\text {a,* }}$, Y.B. Xu ${ }^{\text {a,b }}$, Z.H. Lai ${ }^{\text {c }}$, L.T. Shen ${ }^{\text {b }}$, Y.L. Bai ${ }^{b}$ \\ a State Key Laboratory for Fatigue and Fracture of Materials, Institute of Metal Research, Chinese Academy of Sciences, 75 Wenhua Road, \\ Shenyang 110015, People's Republic of China \\ ${ }^{\mathrm{b}}$ Laboratory for Non-linear Mechanics of Continuous Media, Institute of Mechanics, Chinese Academy of Sciences, \\ Beijing 100080, People's Republic of China \\ ${ }^{\mathrm{c}}$ International Center of Materials Physics, Chinese Academy of Sciences, Shenyang 110015, People's Republic of China
}

Received 7 December 1998; received in revised form 13 February 1999

\begin{abstract}
An investigation has been made into the plastic deformation behavior of a Monel alloy deformed at high strain rate of $10^{5} \mathrm{~s}-1$ by split Hopkinson bar. The results reveal that there are some equiaxed grains with an average size of $150 \mathrm{~nm}$ in diameter in the center of the shear bands, suggesting that this microstructure characteristics be developed by dynamic recrystallization, arising from the deformation and the rapid temperature rise in the band. Analysis shows that the plastic strain rate and the mobile dislocation density play a key role in the new crystallized grain formation and growth. Based on grain boundary energy change and diffusion mechanism, the grain growth kinetics is developed for plastic deformation at a high strain rate. C 2000 Elsevier Science S.A. All rights reserved.
\end{abstract}

Keywords: Dynamic recrystallization; Plastic deformation; High strain rate; Subgrain misorientation

\section{Introduction}

In the past few years, ultra-fine recrystallized grains have been observed in the adiabatic shear bands when materials were deformed to large plastic strains at high strain rates [1-6]. In these literatures, the recrystallization was identified as 'dynamic recrystallization'. Based on Bailey and Hirsh's nucleation mechanism for recrystallization [7], Derby [8-10] developed a theory of dynamic recrystallization and thus he gave a general formula for the grain size and deformation stress. However, Hines and Vecchio [6], through their studies on the recrystallization kinetics within the adiabatic shear bands formed in shock-prestrained copper deformed to high strain at high strain rate, concluded that the observed recrystallized grain sizes in the adiabatic shear bands could hardly be explained in terms of the existing kinetics of the two classical mechanisms of recrystalliza-

\footnotetext{
* Corresponding author. Tel.: + 86-22-27401291; fax: + 86-2227404471.

E-mail address: qli@imr.ac.cn (Q. Li)
}

tion, i.e. high angle boundary migration and subgrain coalescence.

On the other hand, Segal [11], Ferrasse [12] and Valiev et al. [13] used Equal Channel Angular Extrusion (ECAE) to impose simple shear to some materials and got ultra-fine grain structure. The experimental results showed that the intense simple shear during ECAE at ambient temperature surely enhanced dynamic recrystallization.

In this paper, we report the results of dynamic recrystallization in the shear bands of Monel alloy deformed at high strain rate of $4.3 \times 10^{5} \mathrm{~s}^{-1}$. The kinetics of recrystallized grain is examined, and an appropriate model for dynamic recrystallization is proposed.

\section{Experimental procedure}

A modified compression split Hopkinson bar is used to study the dynamic behavior of the alloy. The configuration of the 'hat-shaped' specimen is shown in Fig. 1. Monel alloy with initial grain diameter of $15 \mu \mathrm{m}$ 


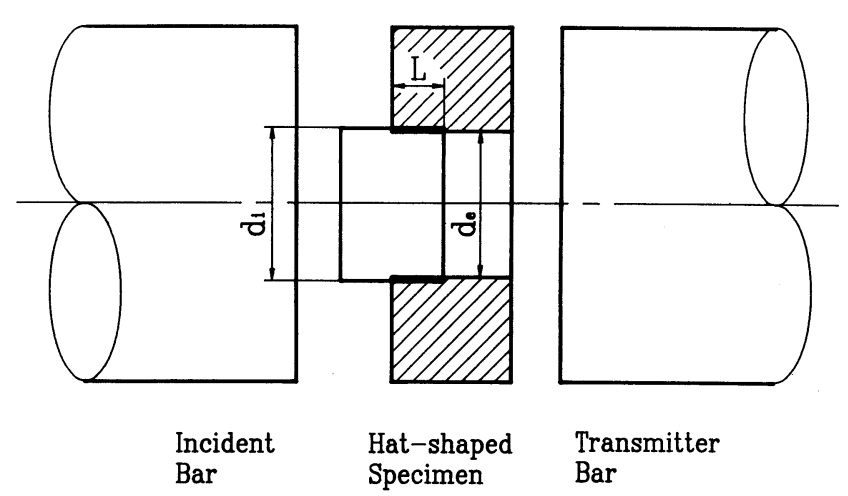

Fig. 1. Profile of a hat-shaped specimen.

used in this investigation has the following compositions (wt.\%)

$\begin{array}{llllllll}\mathrm{Ni} & \mathrm{Cu} & \mathrm{Al} & \mathrm{Ti} & \mathrm{Fe} & \mathrm{Mn} & \mathrm{C} & \mathrm{Si} \\ 65.1 & 29.5 & 3.00 & 0.48 & 0.9 & 0.67 & 0.15 & 0.20\end{array}$

The conventional mechanical and physical properties at room temperature are listed in Table 1. The hatshaped specimen was hit by Hopkinson pressure bar. From the results of Undrade and Meyers, the shear stress $(\tau)$, strain $(\gamma)$ and strain rate $(\dot{\gamma})$ in the shear band are expressed as follows respectively:

$$
\begin{aligned}
& \tau(t)=\frac{E_{0} d_{\mathrm{i}}^{2}}{L\left(d_{\mathrm{i}}+d_{e}\right)} e_{\mathrm{t}}(t) \\
& \dot{\gamma}=\frac{2 c_{0}}{w}\left(e_{t}(t)-e_{\mathrm{t}}(t)\right) \\
& \gamma=\int_{0}^{t} \dot{\gamma} d t
\end{aligned}
$$

where $E_{0}$ and $c_{0}$ are the elastic modulus and elastic wave speed in Hopkinson pressure bar, $L$ and $w$ are the length and width of the shear band ( $w$ is measured on the section plane using metalloscope), $d_{\mathrm{i}}, d_{\mathrm{e}}$ the geometrical parameters of the hat-shaped specimen shown in Fig. $1 ; e_{\mathrm{i}}(t)$ and $e_{\mathrm{t}}(t)$ shown in Fig. 2 are the measured strains of the incident and transmitted stress pulse on the Hopkinson bars, respectively.

The samples for transmission electron microscopy (TEM) were cut from the hat-shaped specimens by electrical discharge machine. After hand-grinding, the sample was reduced to a thickness of $0.150 \mathrm{~mm}$, a dent upon the shear band was made using Gatan dimpler. Finally, the foils were prepared by electropolishing in a solution of 8 pet Nital at $243 \mathrm{~K}$.

Table 1

The conventional mechanical properties of the Monel alloy

\begin{tabular}{llllll}
\hline Yield strength & Tensile strength & Elongation & Reduction & hardness & Density \\
\hline $800 \mathrm{MNm}^{(2}$ & $1100 \mathrm{MN} \mathrm{m}^{(2}$ & $40 \%$ & $20 \%$ & $300 \mathrm{HB}^{3}$ & $8.8 \mathrm{~g} \mathrm{~cm}^{(3}$ \\
\hline
\end{tabular}

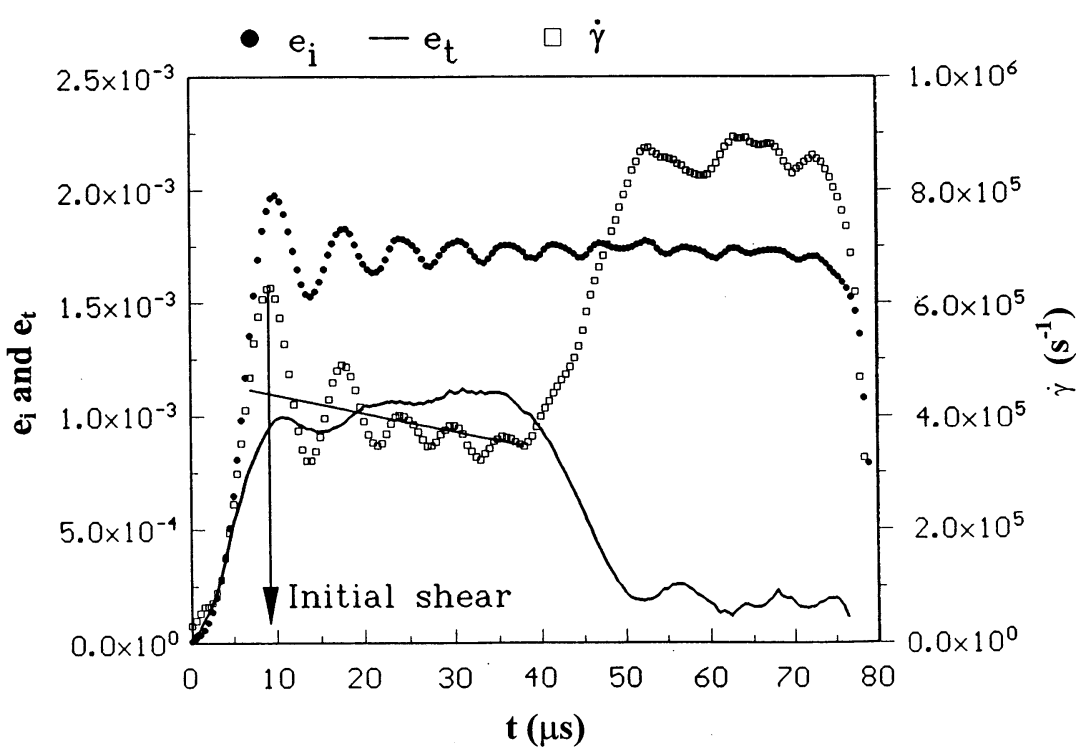

Fig. 2. Variation of the measured strain with the loading time. 


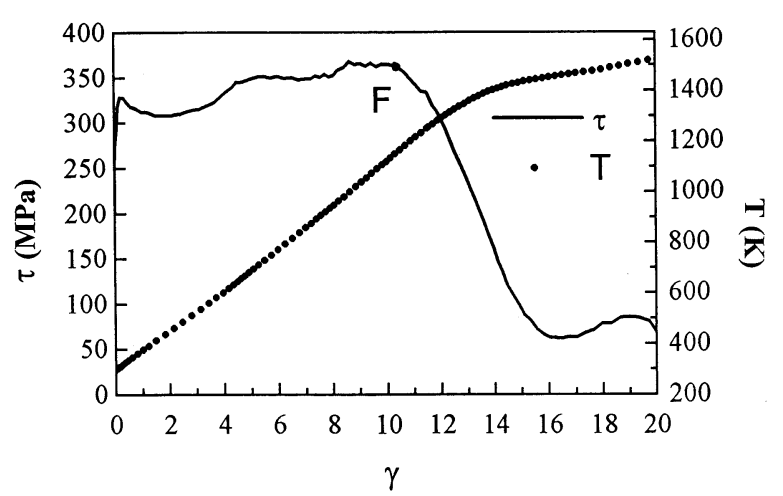

Fig. 3. The shear stress vs. shear strain response and the temperature rise in the shear band during plastic deformation.

\section{Results and discussion}

\subsection{Flow stress-strain response}

When the specimen was loaded by Hopkinson pressure bar, the force applied to the shear band was directly measured by the strain gauges on the incident and transmitted bars, shown in Fig. 2. From Eq. (2) and the time functions of the incident and transmitted pulse strains, the strain rate within the band can be obtained indirectly. Hines and Vecchio [6] have pointed out that the starting time for the shear band formation is just the instant when the strain rate reaches the first vibration peak. Therefore, the relationship of the plastic flow stress to the strain in the shear band can be easily obtained using Eq. (1), Eq. (2) and Eq. (3) and the measured data, this is shown in Fig. 3. Generally, the plot of shear stress vs. strain is divided into two stages. In the first one, the stress is essentially constant, implying that there is a balance between the hardening and the softening in the shear band. In the second stage, the stress decreases sharply with the increasing strain. The reason for this sudden drop is attributed to crack formation in the shear band as shown in Fig. 4.

\subsection{The microstructure within the shear bands}

Observations by TEM reveal clearly the characteristics of the recrystallized grains in the center of the shear band with an average grain size of diameter of $150 \mathrm{~nm}$ and a low density of dislocation as shown in Fig. 5a and Fig. $5 \mathrm{~b}$. Diffraction pattern taken from the center of the shear band indicates that the maximum grain boundary misorientation is approximately $12^{0}[14]$. The pattern also shows that the shear band keeps FCC structure just the same as the matrix. In other words, there is no phase transformation occurring during the high speed plastic deformation.

The microstructure evolution during the plastic deformation is the essential issue of this research topic [15-19]. It is rather difficult to observe directly how the microstructure is evolved in the deformation process. Fortunately, the microstructural characteristics in different parts of the transition region between the band center and the matrix is an indication of the evolutional processes occurring at successive strain stages in the center of the shear band since the strain decreases gradually from the maximum within the band center to zero far in the matrix. Therefore, from the elongated cell structures in the transition region shown in Fig. 6a and slip traces observed in the matrix grains near the band (see Fig. 6b), it is reasonable to deduce the evolution in structure in the band at different strain stage as follows. At the beginning of the plastic deformation in the band, gliding occurs in the favorable slip planes, and a large amount of dislocations is produced between in the slip planes. Thus dislocation boundaries and typical lamellar structure with elongated cells due to the inhomogeneously distributed dislocations appear out. These have also been observed within the shear bands of some other materials such as steels [15-19] and aluminium [20].

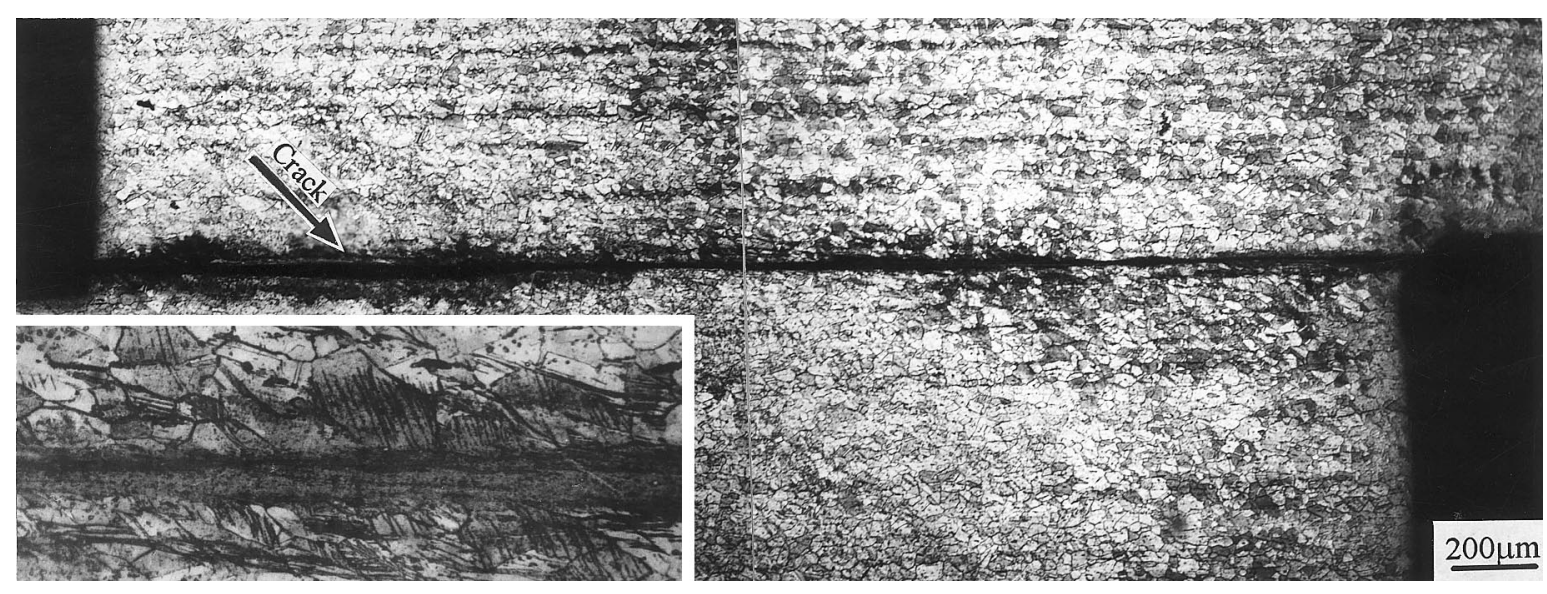

Fig. 4. Shear band obtained in the hated specimen during dynamic impact compression. 

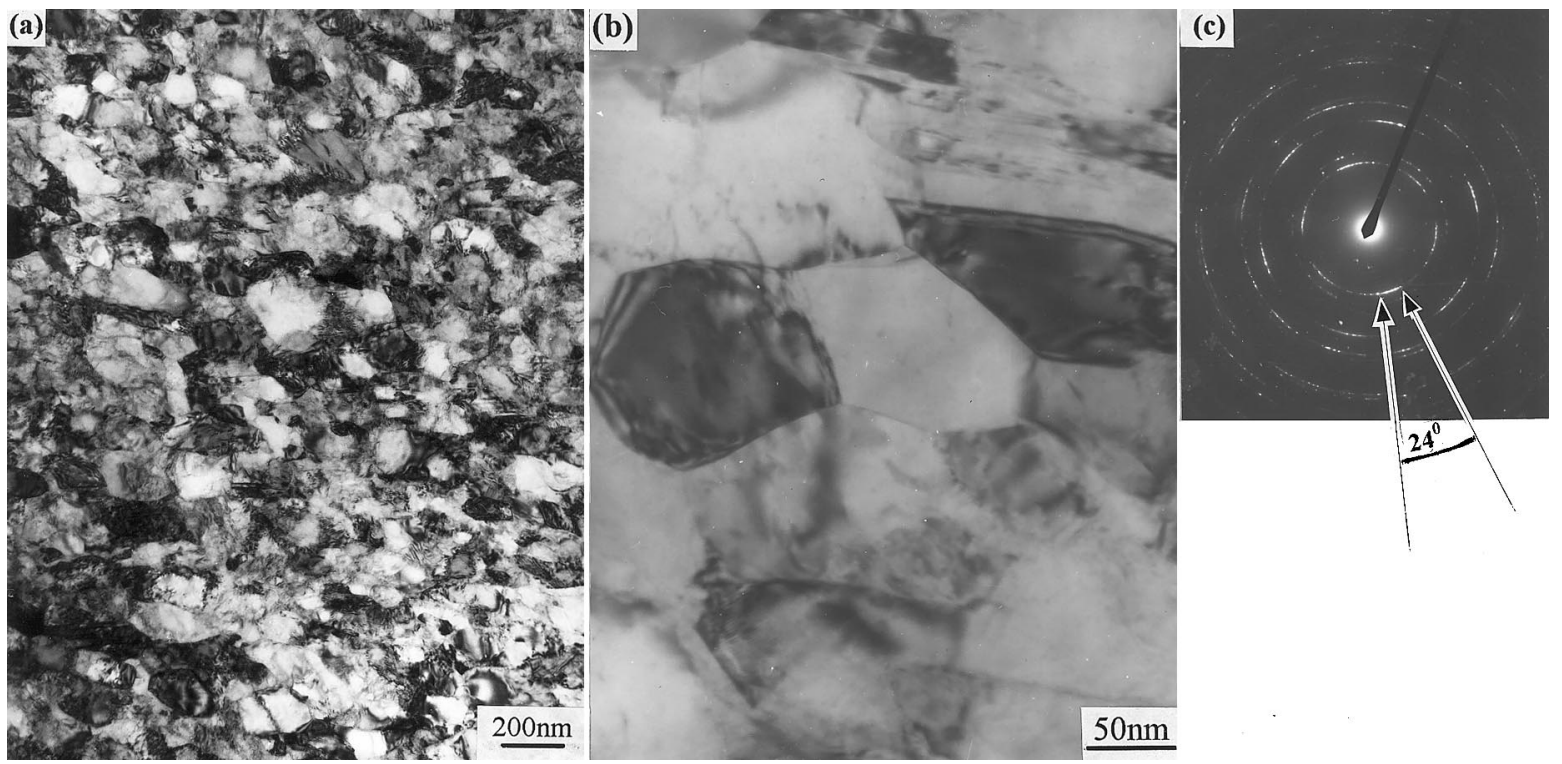

Fig. 5. TEM micrographs showing recrystallized grains within the center of the shear ((a) and (b)) and the diffraction pattern from the center of the shear band (c).

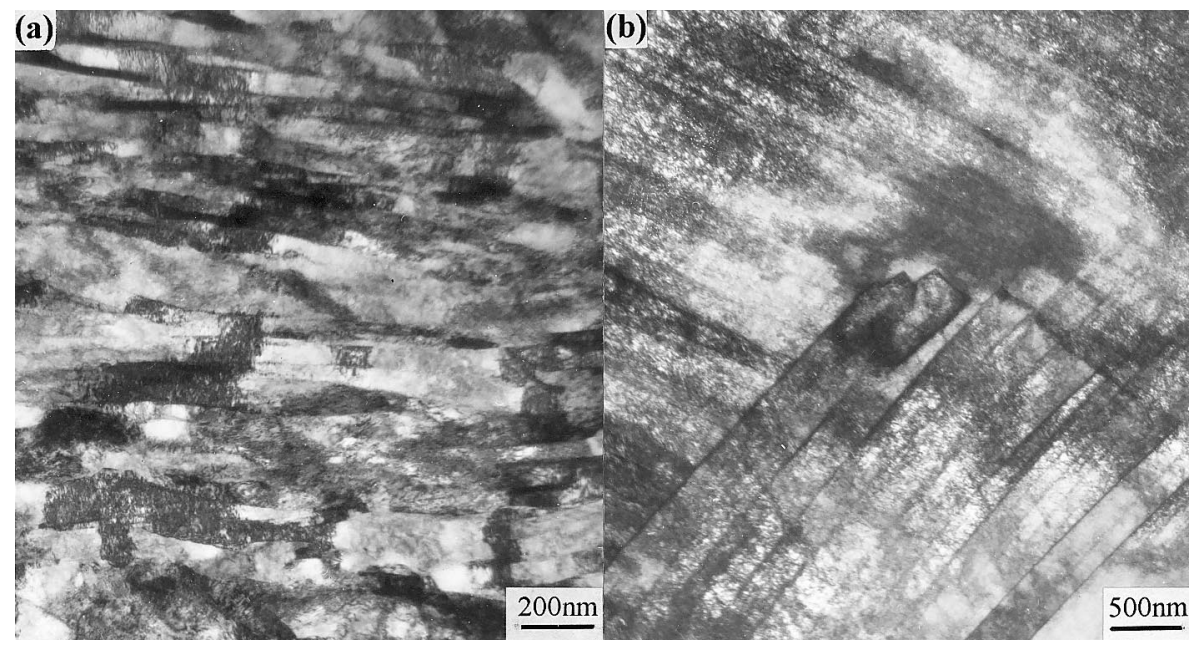

Fig. 6. TEM micrographs in the transition region between the center of the band and the alloy matrix showing the lamellar structure with elongated cells (a) and the slip lines in the matrix very near the band.

\subsection{The kinetics of recrystallization-grain formation and growth}

With increasing strain or strain rate, substantial dislocations are trapped in the cell walls, which make the subgrains rotating to accommodate further deformation [21]. From the energy balance equation, we obtain:

$\frac{d}{d t}\left(\eta \pi D_{1}^{2}\right)=\alpha \mu b^{2} \pi D_{1}^{2} \delta \dot{\rho}_{\mathrm{w}}$

where $\eta$ is the boundary energy density, $D_{1}$ is the equivalent diameter of cell structure (or subgrain); $\alpha \mu b^{2}$, the energy per unit length of dislocation $(\alpha: a$ constant of $0.5 \sim 1.0$ ) [22]; $\delta$, the cell wall width and $\dot{\rho}_{\mathrm{w}}$, the dislocation density rate within the cell wall. $\dot{\rho}_{\mathrm{w}}$ is given by the following expression [21]
$\dot{\rho}_{\mathrm{w}}=\sqrt{\frac{2}{3}\left(\frac{D_{1}}{\delta b}\right) \sqrt{\rho_{\mathrm{m}}} \dot{\gamma}}$

where $\rho_{\mathrm{m}}$ is the mobile dislocation density. Substituting Eq. (5) into Eq. (4), the following expression follows immediately,

$\dot{\eta}+\frac{2 \eta}{D_{1}} \dot{D}_{1}=\alpha \mu b D_{1} \sqrt{\frac{2 \rho_{\mathrm{m}}}{3}} \dot{\gamma}$

From Eq. (6), before the appreciable grain growth occurs, i.e. incubation period, the subgrains will keep their sizes $D_{1}$ essentially unaltered. Then, the subgrains likely begin their rotation as a main mode of deformation. For small misorientation boundary, Derby [9] derived the relation $\eta=\mu b \theta$, where $\theta$ is the subgrain boundary misorientation angle. Putting the $D_{1}=$ con- 
stant in Eq. (6), the kinetics for the subgrain rotation turn out to be:

$\dot{\theta}=\alpha D_{1} \sqrt{\frac{2 \rho_{\mathrm{m}}}{3}} \dot{\gamma} \quad$ or $\frac{d \theta}{d \gamma}=\alpha D_{1} \sqrt{\frac{2 \rho_{\mathrm{m}}}{3}}$

That is to say that the rotational rate is determined by $\rho_{m} \dot{\gamma}$ and that the subgrain boundary misorientation angle increases continuously with the strain until the misorientation angle is of such value that the boundary becomes easily migratory. In other words, the subgrain with average diameter $D_{1}$ now become the recrystallizing centers capable to grow further with the continuous deformation. Referring to the left hand of Eq. (6), the second term will now dominated over the first term, and so assuming $\eta=$ constant, the grain diameter $\left(D_{2}\right)$ of the recrystallized grains will be a function of strain:

$\dot{D}_{2}=\frac{\alpha \mu b}{2 \eta} D_{2}^{2} \sqrt{\frac{2 \rho_{\mathrm{m}}}{3}} \dot{\gamma} \quad$ or $\frac{d D_{2}}{d \gamma}=\frac{\alpha \mu b}{2 \eta} D_{2}^{2} \sqrt{\frac{2 \rho_{\mathrm{m}}}{3}}$

The solution of the Eq. (8) is

$D_{2}=D_{1}\left(\frac{1}{1-B\left(\gamma-\gamma_{\mathrm{c}}\right) D_{1}}\right), B=\frac{\alpha \mu b}{2 \eta} \sqrt{\frac{2 \rho_{\mathrm{m}}}{3}}$

where $\gamma_{c}$ is the critical strain for the subgrains stopping their rotation and starting migration. This shows that if the recrystallization occurs during the deformation, the grain diameter increases with the strain, i.e. the recrystallized grain diameter increases with time since the strain rate is a constant. This result is consistent with that for large deformation studied by Dunne et al. [23]. Eq. (9) is suitable for the continuous deformation at high strain rate since, once the deformation has ceased, the dislocation density within the grain boundary can be decreased from $10^{12}$ to $10^{13} \mathrm{~cm}^{-2}$ to $10^{8}$ to $10^{9} \mathrm{~cm}^{-2}$ in a very short duration such as $10 \mu$ s [21]. In the case of discontinuous deformation, $\rho_{\mathrm{m}}$ in Eq. (9) is likely to decrease also due to hardening or locking so that $\mathrm{B}$ in Eq. (9) tends to diminish in the later stages of deformation. The combined effect of the diminishing $B$ and the increasing $\gamma$ will counter act the growth of $D_{2}$.

When a metal is subjected to plastic deformation, most of the work done in the deformation process is converted into heat. Thermal energy certainly would have some contributions to the grain growth. First of all, we can evaluate the temperature in the shear band using the following expression, which is otherwise difficult to measure directly.

$$
T=T_{0}+\frac{\beta}{\rho c} \int_{0}^{\gamma} \tau d \gamma
$$

where $T_{0}$ is the test temperature (room temperature: $298 \mathrm{~K}), \beta$ is the fraction of plastic work converted to heat, $\beta \sim 0.9, \rho$ is the mass density, $c$ is the specific heat. From the shear stress-strain curve shown in Fig. 3 and Eq. (10), $T$ is calculated and shown also in Fig. 3.
The temperature in the shear band increases linearly with strain before cracking occurs. Because the strain rate is essentially constant, the temperature varies linearly with the deformation time as well. It is worthy to note that the highest temperature within the band is $1165 \mathrm{~K}$ at the point $F$ on the flow (shear) stress-strain curve (Fig. 3), which corresponds to the band cracking.

At such a high temperature, atomic migration under a certain driving force should be taken into account for grain growth. Valiev et al. [13] formulated the grain growth rate under such circumstance,

$\dot{D}_{3}=\left(D_{\mathrm{B}} / k T\right) F^{\mathrm{p}}$ with $p \geq 0$

where $D_{\mathrm{B}}$ is grain boundary diffusion coefficient, $k$ is Boltzmann's constant, $T$ is the absolute temperature and $F$ is the driving force. Now $D_{3}$ stands for the instantaneous grain size. Since $D_{\mathrm{B}}$ is proportional to vacancy content in the grain boundary, $D_{\mathrm{B}}=C_{\mathrm{V}} D_{\mathrm{B}}^{\mathrm{V}}$ where $C_{\mathrm{v}}$ and $D_{\mathrm{B}}^{\mathrm{V}}$ are vacancy concentration and vacancy diffusion coefficient in the grain boundary, respectively. As dislocations are absorbed into grain boundary, more vacancies are expected to appear in the grain boundary. These excess vacancies increase the grain growth rate as can be seen from Eq. (11) [24]. Clark and Alden [25] have further proposed an 'excess' vacancy model for the interpretation of deformationenhanced grain growth during superplastic deformation expressed as follows:

$D_{\mathrm{B}}=D_{\mathrm{B}_{0}}+D_{\mathrm{B}}^{\mathrm{V}} \chi_{\varepsilon}$

$\chi_{\varepsilon}=k_{1} \dot{\gamma}[1-\exp (-t / \omega)]$

where $D_{\mathrm{B}_{0}}$ is the usual grain boundary diffusion coefficient, $k_{1}$ is a constant, $\omega$ is the vacancy relaxation time. In the case of the high speed deformation, $t<<\omega$ we may obtain the following expression

$\chi_{\varepsilon}=k_{1} \dot{\gamma} t$

On the other hand, Valiev [13] has given that the driving force is

$F=(\Omega / \delta)\left(2 \eta / D_{3}+2 \tau_{\mathrm{i}}^{2} / E\right)$

$\Omega=b^{3}$ is the atomic volume, $\tau_{\mathrm{i}}$ is the applied shear stress, $E$ is the elastic modulus.

Substituting Eq. (12), Eq. (14) and Eq. (15) into Eq. (11) and taking $p=1$ for simplicity[13], we obtain the grain growth rate if we consider the thermal grain growth alone,

$\frac{d D_{3}}{d t}=\left(D_{\mathrm{B}_{0}}+k_{1} \gamma D_{\mathrm{B}}^{\mathrm{V}} t\right)\left(\frac{2 \eta}{D_{3}}+\frac{2 \tau_{\mathrm{i}}^{2}}{\mathrm{E}}\right) \frac{\Omega}{\delta k T}$

$D_{\mathrm{B}_{0}}=D_{0}^{\prime} \exp \left(-Q_{\mathrm{B}} / R T\right)$

$D_{\mathrm{B}}^{\mathrm{V}}=D_{0}^{\prime \prime} \exp \left(-Q_{\mathrm{B}} / R T\right)$

where $D_{0}^{\prime}$ and $D_{0}^{\prime \prime}$ are the preexponential factors, $Q_{\mathrm{B}}$ is the activation energy of grain boundary diffusion, $Q_{\mathrm{B}}^{\mathrm{V}}$ is 
Table 2

Relevant parameters of tested materials

\begin{tabular}{llllllllllll}
\hline$b$ & $\begin{array}{l}D_{0}^{\prime} \\
\left.\left(10^{-10}\right) \mathrm{m}\right)\end{array}$ & $\begin{array}{l}Q_{\mathrm{B}} \\
\left(\mathrm{m}^{2} \mathrm{~s}^{-1}\right)\end{array}$ & $\begin{array}{l}Q_{\mathrm{B}}^{\mathrm{V}} \\
\left(\mathrm{kJ} \mathrm{mol}^{-1}\right)\end{array}$ & $\begin{array}{l}E \\
(\mathrm{GPa})\end{array}$ & $\begin{array}{l}\mu \\
(\mathrm{GPa})\end{array}$ & $\begin{array}{l}c \\
\left(\mathrm{~J} \mathrm{~kg} \mathrm{k}^{-1} \mathrm{k}^{-1}\right)\end{array}$ & $\begin{array}{l}\eta \\
\left(\mathrm{J} \mathrm{m}^{-2}\right)\end{array}$ & $\begin{array}{l}k_{1} \\
{[24]}\end{array}$ & $\begin{array}{l}\tau_{i} \\
(\mathrm{MPa})\end{array}$ & $\begin{array}{l}\dot{\gamma} \\
\left(\mathrm{s}^{-1}\right)\end{array}$ & $\begin{array}{l}\delta \\
(\mathrm{m})\end{array}$ \\
\hline 2.56 & $\begin{array}{l}1.37 \\
\times 10^{-5}[9]\end{array}$ & $115[9]$ & $77.2[26]$ & 180 & 65.5 & 419 & 1.46 & 0.00041 & 350 & $4.3 \times 10^{5}$ & $10^{-9}$ \\
\hline
\end{tabular}

the activation energy of grain boundary vacancy migration and $R$ is the gas constant. Since the excess vacancies are created by dislocation annihilation within grain boundaries, the thermal activation of the vacancy formation is not necessary in the boundary and we need only to consider the migration of vacancies. So it is plausible to assume $D_{0}^{\prime}=D_{0}^{\prime \prime}$.

So far, we have separately formulated the effect of high strain rate and the atomic thermal migration on the kinetics of the recrystallization. The former is an athermal process, and the later is obviously temperature dependent. It is plausible to superimpose simply these two effects, and then the grain growth rate could be expressed by the following differential equation from the Eq. (16a) and Eq. (9)

$\frac{d D}{d t}=\left(D_{\mathrm{B}_{0}}+k_{1} \dot{\gamma} D_{\mathrm{B}}^{\mathrm{V}} t\right)\left(\frac{\eta}{D}+\frac{\tau_{\mathrm{i}}^{2}}{E}\right) \frac{2 \Omega}{\delta k T}+\frac{\alpha \mu b}{2 \eta} D^{2} \sqrt{\frac{2 \rho_{\mathrm{m}}}{3}} \dot{\gamma}$

This equation could be solved only using numerical method by means of a computer. In order to solve this differential equation, the initially growing recrystallized grain size (i.e. $D_{1}$ in Eq. (7)), the critical strain $\left(\gamma_{c}\right)$ for the grain starting to growth and the mobile dislocation density $\rho_{\mathrm{m}}$ have to be known. Clifton [27] has given that the mobile dislocation density $\rho_{\mathrm{m}}=2.4 \times 10^{12} \mathrm{~m}^{-2}$ at a strain rate $\dot{\gamma}=2.4 \times 10^{5} \mathrm{~s}^{-1}$ for pure metals. The mobile dislocation density in alloys is usually less than that in pure metals due to the much more blockades in an alloy. Therefore, as a working example, we are plausible to take the mobile dislocation density in Monel alloy as $\rho_{\mathrm{m}}=$ $10^{11}$ and $10^{10} \mathrm{~m}^{-2}$ and assume $\gamma_{\mathrm{c}}=4.3$ (the reason for this value is given elsewhere) and three values of recrystallized initial grain diameter $D_{1}=30,50,70 \mathrm{~nm}$. The values for $D_{0}^{\prime}, Q_{\mathrm{B}}$ and $Q_{\mathrm{B}}^{\mathrm{V}}$ are all taken from those of pure nickel since Monel alloy is a nickel based alloy. Other relevant constants used in the solution of Eq. (17) are listed in Table 2. The calculated results are shown in Fig. 7. It can be found that the grain diameter $(D)$ increases almost linearly with the time $(t)$, and then exponentially increases with the time after several microseconds. From Fig. 3, it is seen that the final strain in the shear band of Monel alloy is about 10. In this example, the recrystallized grain has grown for $14 \mu$ s from the beginning of dynamic recrystallization to the end of the deformation and the grain size finally reaches $130 \sim 200 \mathrm{~nm}$, see Fig. 6. This is quite in accord with the observation by TEM.

\section{Conclusions}

1. The recrystallized grains and elongated subgrains are observed within the shear bands of Monel alloy, which is deformed to a large strain at high strain rate of $4.3 \times 10^{5} \mathrm{~s}^{-1}$.

2. The microstructures in the transition region between the shear band and the matrix is the typical lamellar structure and elongated cells. This suggests that the microstructure in the band undergoes successively the dislocation multiplication, inhomogeneous distribution, the grain subdivision by dislocation boundaries, and the subgrain rotation during the plastic deformation. The subgrain rotation rate is proportional to the strain rate. The recrystallized grains start from those subgrains having separated by high angle migratory grain boundaries.

3. Based on the grain energy change and diffusion mechanism, the grain growth kinetics is developed concerning the high speed plastic deformation. The analysis shows that the plastic strain rate and the density of mobile dislocation play the key role in the new recrystallized grain formation and growth during the plastic deformation. If the temperature rise in the shear band is high enough due to adiabatic heating, the recrystallized grain growth is also controlled by atomic thermal migration. In this process, the strain rate effect is still noticeable.

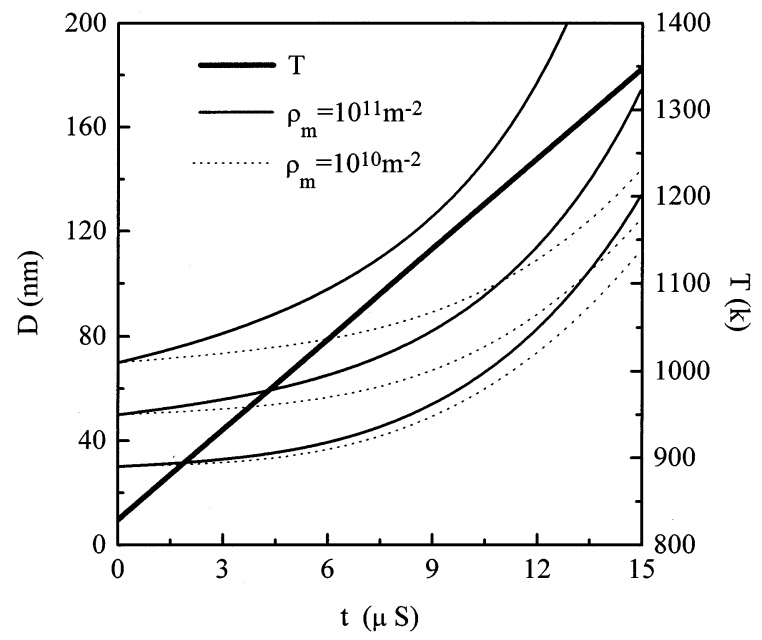

Fig. 7. Variation of the recrystallized grain size $(D)$ with the deformation time $(t)$, the thin line representing $\rho_{\mathrm{m}}=10^{11} \mathrm{~m}^{-2}$, the dot line representing $\rho_{\mathrm{m}}=10^{10} \mathrm{~m}^{-2}$ and the coarse line denoting the temperature in the band. 


\section{References}

[1] M.A. Meyers, H.-R. Pak, Acta Metall. Mater. 34 (1986) 2493 2499.

[2] A.H. Chokshi, M.A. Meyers, Scripta Metall. Mater. 24 (1990) 605-610.

[3] U. Andrade, M.A. Meyers, et al., Acta Metall. Mater. 42 (1994) 3183-3193.

[4] M.A. Meyers, Y. -J. Chen, et al., Met. Mater. Trans. 26A (1995) 2493-2501.

[5] Q. Li, C.X. Ma, Z.H. Lai, Chin. J. Mater. Res. (in Chinese) 11 (1997) 42-46.

[6] J.A. Hines, K.S. Vecchio, Acta Metall. Mater. 45 (1997) 635 649.

[7] J.E. Bailey, P.B. Hirsch, Proc. Roy. Soc. 267 (1962) 11-30.

[8] B. Derby, M.F. Ashby, Scripta Metall. Mater. 21 (1987) 879884.

[9] B. Derby, Acta Metall. Mater. 39 (1991) 955-962.

[10] B. Derby, Scripta Metall. Mater. 27 (1992) 1581-1586.

[11] V.M. Segal, Mater. Sci. Eng. A197 (1995) 157-164.

[12] S. Ferrasse, V.M. Segal, et al., Met. Mater. Trans. 28A (1997) $1047-1057$.

[13] R.Z. Valiev, E.V. Kozlov, et al., Acta Metall. Mater. 42 (1994) $2467-2475$.
[14] Y. Iwahashi, Z. Horita, et al., Acta Metall. Mater. 45 (1997) $4733-4741$.

[15] Y.B. Xu, Z.G. Wang, Mater. Sci. Eng. 114A (1989) 81-87.

[16] Y.B. Xu, Y.L. Bai, et al., Acta Metall. Mater. 44 (1996) 19171926.

[17] K.M. Cho, S. Lee, S.R. Nutt, J. Duffy, Acta Metall. Mater. 41 (1993) 923-932.

[18] K. Cho, Y.C. Chi, J. Duffy, Metall. Trans. 21A (1990) 11611175.

[19] J. Krejci, J. Brzina, J. Buchar, Scripta Metall. Mater. 27 (1992) 611-616.

[20] D.A. Hughes, N. Hansen, Acta Metall. Mater. 45 (1997) $3871-$ 3886.

[21] J.A. Hines, K.S. Vecchio, S. Ahzi, Met. Mater. Trans. 29A (1998) 191-203.

[22] J.P. Hirth, J. Lothe, Theory of Dislocations, McGraw-Hill, New York, 1968.

[23] F.R.E. Dunne, M.M. Nanneh, M. Zhou, Phil. Mag. 75A (1997) $587-610$.

[24] D.J. Sherwood, C.H. Hamilton, Phil. Mag. 70A (1994) 109-143.

[25] M.A. Clark, T.H. Alden, Acta Metall. Mater. 21 (1973) 11951206.

[26] H. Mughrabi, A. Seeger, Phys. Stat. Sol. 19 (1967) 251-262.

[27] R.J. Clifton, J. Appl. Mech. 50 (1983) 941-952. 